Instituto Internacional de Investigación y Desarrollo Tecnológico Educativo INDTEC, C.A.

DOI: https://doi.org/10.29394/scientific.issn.2542-2987.2016.1.1.14.249-265

OAI-PMH: http://www.indteca.com/ojs/index.php/Revista Scientific/oai

\title{
Formación del Docente y Pedagogía de la Complejidad en el Contexto de la Educación
}

\author{
Autora: Naylet Chiquinquirá Rojas Rivero \\ Universidad Fermín Toro, UFT \\ nayletrojas@hotmail.com; nayletrojas3@gmail.com \\ Barquisimeto, Venezuela
}

\section{Resumen}

La naturaleza del acto pedagógico en el contexto de la Educación, comporta una complejidad relacional sistémica que compromete la formación y la identidad profesional del docente. En este sentido, la presente investigación se propone como objetivo fundamental, la formación del docente y pedagogía de la complejidad en el contexto de la Educación. La investigación se fundamenta epistemológicamente en los postulados del pensamiento complejo, el cual según Morín (2001), es un pensamiento que postula la dialógica, la recursividad y la hologramaticidad como principios pertinentes. El modelo de investigación se corresponde con el paradigma cualitativointerpretativo propuesto por Carr y Kemmis (1998), el cual está orientado a interpretar las estructuras de significados subjetivos que construyen los actores sociales acerca de la realidad, lo cual se logrará con apoyo en el método fenomenológico-hermenéutico, utilizando como técnica de recolección de la información la entrevista en profundidad.

Palabras clave: formación; docente; pedagogía de la complejidad; educación. 


\title{
Teacher Training and Education of Complexity in the Context of Education
}

\begin{abstract}
The nature of the pedagogical act in the context of education, involves a systemic relational complexity that involves training and professional identity of teachers. In this sense, this research is proposed as a fundamental objective, teacher training and pedagogy of complexity in the context of Education. The research is based epistemologically on the principles of complex thought, which according to Morin (2001), is a thought that posits the dialogical, recursion and hologramaticidad as relevant principles. The research model corresponds to the qualitative-interpretive paradigm proposed by Carr and Kemmis (1998), which is geared to interpret the structures of subjective meanings that social actors about reality, which will be achieved through support in the phenomenological hermeneutical method, using as a technique for data collection in-depth interview.
\end{abstract}

Keywords: training; teaching; education complexity; education. 


\section{Introducción}

El devenir histórico de la humanidad en el campo de la construcción del conocimiento, nos muestra el interés del ser humano por descubrir los diversos fenómenos implicados en la interacción entre sujeto, realidad, procesos sociales y culturales, lo cual siempre ha sido objeto de preocupación por parte de los actores de la ciencia. En este sentido, los avances que se han logrado en el campo de las Ciencias de la Educación, también han sido producto de un esfuerzo científico y sistemático de creación, dado que la educación como meta proceso dinámica, está llamada a generar grandes cambios sociales, donde cada individuo tiene la oportunidad de desarrollar al máximo sus capacidades y potencialidades.

Por lo tanto, cualquier transformación que se pretenda llevar a cabo en un determinado campo de la educación, requiere, no solamente de recursos humanos debidamente motivados y capacitados para emprender la transformación que se desea alcanzar, sino de una ruptura de los viejos modelos de pensamiento, que habilite a los actores para encarar los grandes desafíos que plantean las interproblemáticas de los contextos educativos, políticos y culturales de una sociedad mundo caracterizada por la incertidumbre.

Ante estos señalamientos, Morín (2002, pág. 24), sostiene que "la educación debe favorecer la aptitud natural del pensamiento para plantear y resolver los problemas $\mathrm{y}$, correlativamente, estimular el empleo de la inteligencia general". De lo expresado por este autor, se desprende, que a pesar de los grandes aportes hechos por diversos pensadores $\mathrm{e}$ investigadores al campo de la educación, aún sus particularidades de integración, de interdisciplinariedad, de complejidad y dinamismo, siguen nutriendo curiosidades en la búsqueda de nuevos procedimientos educativos para renovar el proceso de enseñanza-aprendizaje de los educandos. 
Actualmente, al profesional de la docencia se le cuestionan viejas concepciones de enseñanzas, que en cierta medida, no toma en cuenta los valores sociales del aprendizaje, en tanto su praxis muchas veces se limita a transmitir conocimientos, sin considerar la participación y autonomía del alumno, así como también la creatividad y la innovación en el acto pedagógico. Sobre este particular Pérez (2000) refiere:

Es urgente que los educadores introyectemos y asumamos la necesidad de un cambio profundo y nos aboquemos a gestar una educación que privilegie el aprendizaje autónomo, personal y permanente, la curiosidad, la creatividad, la innovación, la reflexión, la capacidad crítica, el trabajo en equipo, la formación de la persona y la convivencia humana en la solidaridad y el servicio; una educación que enraizada en el hoy de los alumnos y de la vida, capacite para construir un mejor mañana para todos (pág. 18).

Este planteamiento remite a considerar la definición de un nuevo profesional de la docencia, un educador que abandone toda pretensión erudicionista y enciclopedista; además, su gestión debe orientarse al fomento de los procesos superiores del pensamiento, de hacer respetar las diferencias individuales de sus alumnos, en base a cualquier aspecto de su personalidad, y estar capacitado para enfrentar los nuevos retos educativos que la sociedad en la actualidad requiere. Esto significa, que la educación que demanda la sociedad de este tiempo, debe ser pensada desde las universidades para transformar la cultura profesional de los centros escolares, en una nueva cultura que permita la reflexión colectiva y la discusión permanente de los principios que guían la práctica del docente mediador de conocimientos.

Dentro de ese proceso de cambios que hoy deben abordar las instituciones universitarias, se encuentra la formación del docente, por ello, al considerar los contenidos curriculares que se vienen utilizando en la formación de éste profesional, se plantean nuevos desafíos para formar profesionales consustanciados con las realidades de cada sector educativo y a su vez 
debidamente dotados de competencias cognoscitivas e investigativas acordes a las exigencias de los tiempos actuales.

Por otra parte, la introducción de nuevas políticas educativas en el Subsistema de Educación, requiere la aplicación de perspectivas epistemológicas y metodológicas emergentes, para encausar nuevas reorientaciones de los objetivos de la enseñanza aprendizaje del educando, puesto que la función del educador en la empresa educativa, tal y como la refiere Dewey (2002), es "proporcionar un ambiente de aprendizaje renovador que estimule las repuestas y dirija el curso del alumno". (pág. 158).

Ahora bien, todos estos planteamientos conducen a un repensamiento de la formación del docente para encarar la complejidad de la pedagogía en el contexto de la Educación. En este caso se requiere una formación docente, tal y como la visiona Márquez Pérez (2009):

Un proceso que abarque el desarrollo de acciones tendentes a fortalecer la relación entre el saber y la práctica, tanto en profesionales como en aprendices que se ubican en una determinada área del saber...la idea es desarrollar una práctica pedagógica que garantice la efectividad del proceso enseñanza-aprendizaje, haciendo énfasis en una visión integral del ser humano que conjugue: lo social, afectivo, cognitivo y cultural (pág. 36).

Esto traduce la necesidad de pensar la pedagogía desde la perspectiva epistemológica de la complejidad, como única vía para poder lograr la construcción de aprendizajes integrales, pues la complejidad según Morín (2003), "está contra el aislamiento de los objetos del conocimiento; reponiéndolos en su contexto, y de ser posible, en la globalidad en la que pertenecen". (pág. 72).

En este sentido, el propósito general del presente artículo, es analizar la formación del docente y la pedagogía de la complejidad en el contexto de la Educación, asumiendo como modelo investigativo el paradigma cualitativo- 
interpretativo, a los fines de describir las categorías implícitas en el modelo actual de formación del docente, utilizado en las instituciones universitaria e interpretar la praxis pedagógica que desarrollan los docentes de educación en las Instituciones educativas.

\subsection{Formación Docente y Pedagogía Integradora desde el Pensamiento Complejo}

Toda formación académica y pedagógica para generar cambios en el futuro profesional de la docencia, induce a introducirse en el plano de la compleja interacción objetiva y subjetiva del proceso de enseñanzaaprendizaje del estudiante-docente de educación, lo cual conlleva a que en dicho proceso se dé una interacción indisoluble. Esto se debe a que el desarrollo de una destreza, conlleva a favorecer la formación de otras más complejas, dominando así distintos procedimientos y competencias que le ayuden a asumir actitudes renovadoras dentro del proceso formativo que se requiere.

De manera, que el docente formador debe estar consciente de su alto nivel de responsabilidad para instruir y mediar en el aprendizaje, pues en la actualidad hay urgencia de emprender nuevos caminos que se traduzcan en una serie de acciones tendentes a lograr cambios significativos en la conducta del educando. Esto traduce, que el docente debe ser un investigador, un innovador y un creador de nuevas situaciones de aprendizaje, por lo que Ugas (2003), señala que:

El acto pedagógico debe ser entendido como una operación de carácter heurístico, por lo tanto, el maestro debe asumirse como un organizador de saberes, no un transmisor de conocimientos, lo cual significa asumir nuevos procedimientos y protocolos mediante una reorganización permanente de la pedagogía (pág. 142). 
De esto se deduce, que las acciones pedagógicas del docente siguen siendo factores determinantes para modificar conductas en el individuo y generar nuevas situaciones o experiencias de aprendizajes cónsonas con su motivación y capacidad, durante el desarrollo del proceso educativo del educando. Además, cada contexto social posee un ideal educativo que se refleja en los objetivos asignados por la educación que recibe y por el modo en que se constituya y se desarrolle dicho proceso educativo.

En relación con estas ideas, Rozas (2005, pág. 62) expresa que:

El desarrollo de toda profesión está vinculada a la producción de conocimiento, lo cual siempre está referido a los fenómenos sociales que expresan el movimiento de lo real, a lo que debería agregarse la necesidad de que estos conocimientos sean la base de la intervención, ya que los procesos sociales son sumamente complejos y se necesita de la investigación como herramienta para desentrañar e interpelarlos.

Por lo tanto, se requiere un profesional de la docencia capaz de identificar problemas, investigarlos desde una nueva lógica de interrogación y presentar soluciones a los mismos; también se requiere realizar acciones necesarias para que las soluciones sean posibles; y ante estas acciones el profesional de la docencia en educación, juega un papel decisivo frente a los nuevos retos que se presentan, tanto en las aulas de clases como en el contexto de la vida cotidiana. Es por ello, que una de las demandas básicas de la sociedad, es la de contar con profesionales capacitados y eficientemente preparados para enfrentar el desafío de educar desde la unidad y la diversidad, pero también desde la certeza y la incertidumbre.

Siguiendo este mismo discurso, Morín (2003), comenta que el paradigma de la complejidad se sustenta en ciertos principios que lo definen y lo constituye; tales como el principio dialógico, que permite mantener la dualidad en el seno de la unidad del conocimiento; el principio de la recursividad organizacional, que denota un proceso en el cual los productos y 
los efectos son al mismo tiempo, causas y generadores de aquello que lo produce; donde se incluye la totalidad de la información del objeto presentado, y que no sólo las partes están en el todo, sino que el todo está en las partes.

Ante esas exigencias, el profesional de la docencia en Educación, está en la necesidad de reflexionar sobre su ejercicio docente, y es conveniente que se plantee cuestiones como el medio institucional, las influencias del contexto, la formación curricular, y el rol social de las instituciones educativas en general, puesto que casi siempre estos componentes de la realidad no suelen ser valorados y comprendidos para la formación del docente y en especial los modelos de formación que vienen a cultivar el pensamiento y las acciones reflexivas del docente.

\subsection{Formación Docente}

La formación humana es el eje fundamental para la transformación del individuo y de la sociedad, de la cual también forman parte los estudiantes, educadores e instituciones educativas, entre otros actores involucrados en el proceso de interaprendizaje sin embargo, el proceso de globalización existente está repercutiendo en la cultura y en la educación, por lo que se considera urgente y necesario que las instituciones educativas y sus actores redefinan y asuman sus funciones prioritarias. Esto implica retomar desde una perspectiva crítica uno de los objetivos primordiales de la institución universitaria: formar de modo integral el talento humano, lo que representa un desafío permanente y una dimensión central del sector educativo y de la sociedad. La formación del docente, en este sentido, debe orientarse hacia una formación integral, la cual debe ser asumida responsablemente, no sólo por el ser humano sino también por la Institución Universitaria y por el Estado. En este sentido, es necesario revisar las leyes venezolanas que contemplan el derecho a la educación y específicamente, el derecho a la formación del ser humano y a la 
formación del docente universitario, lo cual también es un deber social del Estado.

\subsection{La educación desde una perspectiva humana}

La educación, en su sentido más amplio, juega un papel preponderante, porque ella constituye uno de los instrumentos más poderosos para generar transformaciones en la sociedad. Uno de los desafíos más difíciles será el de modificar nuestro pensamiento de manera que enfrente la complejidad creciente, la rapidez de los cambios y lo imprevisible que caracteriza nuestro mundo, por lo que debemos reconsiderar la organización del conocimiento, derribar las barreras tradicionales entre las disciplinas y concebir la manera de volver a unir lo que hasta ahora ha estado separado. Esto implica reformular las políticas y programas educativos, manteniendo la mirada fija hacia el largo plazo, hacia el mundo de las generaciones futuras frente a las cuales tenemos una enorme responsabilidad (Morín, 2000).

Todos los actores involucrados en el proceso de interaprendizaje deben ser cada día más receptivos a la necesidad de cambios en su formación y práctica educativa. Para que estos cambios se generen es indispensable repensar los paradigmas respecto a lo que es enseñar y aprender: de una concepción de la enseñanza y aprendizaje como transmisión de conocimientos, a otra en la cual el centro de interés de la docencia tenga como condiciones previas:

1. El descontento con nuestras creencias pasadas.

2. Encontrar alternativas inteligibles y útiles.

3. Ver la conexión entre las nuevas creencias y la praxis anterior.

En este orden de ideas, Fairstein, H. (2003). Cuestiona a la educación tradicional de su época, haciendo referencia a la concepción bancaria de la educación. Desde esta concepción, el profesor lo sabe y lo puede todo, 
mientras que el alumno no sabe nada y es totalmente dependiente. Aunque de estas consideraciones han pasado a la rigidez de estas posiciones que niegan a la educación como proceso de búsqueda del conocimiento, aún se mantienen presentes en el sistema educativo actual.

Fairstein, H. (op.cit.) sostiene que "la educación se debe basar en la manera como el alumno se ve y siente el mundo a su alrededor" (p. 20). Desde esta nueva concepción educar es dialogar y el diálogo es un acto de amor, y el maestro es un observador de procesos que no impone valores, sino que observa a sus alumnos a partir de sus expresiones, pensamientos, sentimientos, comportamientos e interacciones.

Otros autores (Corrales, 2008; Flórez, 2001; Osorio Lazo, 1969; Barroso, 1991; Morín, 2000; Figueroa, 2000) coinciden en replantear el propósito de la educación desde una perspectiva más humana, la cual debe estar orientada a contribuir en el desarrollo humano integral de los actores involucrados en el proceso socioeducativo.

Corrales (2008) sostiene que tradicionalmente, "el educador es el poseedor de los conocimientos y el estudiante es un recipiente pasivo de los mismos" (pág. 24); por lo que también destaca que "educación significa poder y control de la información, y que en ella el énfasis es sobre los comportamientos y los contenidos más que sobre los procesos de la relación educativa" (pág. 25). Los maestros son percibidos como controladores, la relación es de miedo, la confianza es mínima y no existe un lugar para el ser humano, sólo se destaca la inteligencia o la razón.

Corrales (op.cit.) propone el modelo de "relación persona-persona, el cual se desarrolla en un clima de mutua confianza, aceptación y mucha apertura, que facilita el aprendizaje" (pág. 28). Desde esta concepción, el estudiante asume responsabilidades en sus procesos y el maestro proporciona recursos y el modelaje desde su propia experiencia. De esta forma, el estudiante desarrolla su programa (solo o en complementación con otros) en 
disciplinas académicas que se derivan de la interacción estudiante-estudiante y estudiante-contexto, y la evaluación es realizada por el mismo estudiante y por el grupo, mediante procesos de retroalimentación. Todos estos aspectos hacen que el aprendizaje sea más profundo, así como más influyente en las vidas y comportamientos de los educandos.

De acuerdo con lo anterior, la educación actual debe repensar su concepción del conocimiento y los métodos de enseñanza-aprendizaje. La nueva orientación de la educación, más que hacer énfasis sólo en contenidos académicos o en una transmisión rígida de saberes, debe centrarse más en el desarrollo integral del ser humano, en fomentar una formación humana integral, proporcionando la oportunidad de hacer de él una persona capaz, digna, crítica, libre.

La educación, en palabras de Fernando Savater, citado en Flórez (2001), "es el más humano y más humanizador de los empeños, con lo cual manifiesta la relevancia e importancia social de quienes practican la docencia" (pág. 36), que son personas que dignifican y se dignifican en el desarrollo de la función cognitiva del permanente acto educativo: el proceso de enseñanzaaprendizaje.

El propósito de la educación, como lo afirma Orozco, L. (2002), citado en Barroso (1991), "es el ser humano y no la asignatura, por lo que la educación debe fortalecer la personalidad del educando y no debilitarla, debe buscar que cada estudiante abandone las aulas, sintiéndose más digno y satisfecho" (pág. 45). La educación como proceso integral debe reforzar el sentido de valoración personal, las capacidades individuales y la definición de contextos integrados, permitiendo nuevas posibilidades en cada quien y la oportunidad de descubrir recursos, alternativas, un mundo propio y personal (Balza, 2009, pág. 46). 


\section{Metodología}

En este apartado se describe la perspectiva epistemológica del estudio, el método en el cual se fundamenta, el tipo y diseño de la investigación, el escenario donde se realizará la investigación, la participación de los informantes claves, las unidades y categorías preliminares del estudio, las técnicas e instrumentos a utilizar para la recolección de la información, y finalmente los criterios de cientificidad del trabajo en desarrollo.

La metódica de trabajo para el desarrollo del presente, se deriva del modelo paradigmático que sustenta la misma. De este modo, el paradigma del pensamiento complejo adoptado como perspectiva de análisis para la reflexión epistémica, demanda de un método que permita superar las insuficiencias de la racionalidad positivista clásica que ha privilegiado, defendido y propugnado la objetividad del conocimiento. Es preciso enfatizar, que para abordar a profundidad el objeto de estudio antes referido, la herramienta metodológica más apropiada es la fenomenología hermenéutica.

Ciertamente, para Sandin (2003), "la tradición fenomenológicahermenéutica o enfoque interpretativo es ontológica, una forma de existir, de ser y estar en el mundo, donde la dimensión fundamental de la conciencia humana es histórica y sociocultural, y se expresa a través del lenguaje" (pág. $63)$.

En tanto para Martínez (2000), "la hermenéutica tiene como misión descubrir el significado de las cosas, interpretar lo mejor posible los escritos, los textos, pero conservando su singularidad en el contexto del que forma parte" (pág. 32).

\section{Conclusiones finales}

Al entrar en el campo de las conclusiones del estudio se inicia tomando en cuenta las inquietudes ontológicas y epistemológicas relacionadas con la formación del docente y aunado también al ámbito referencial interno de los 
diferentes actores que formaron parte del trabajo investigativo (docentes formadores universitarios y docentes de instituciones); por lo tanto, fue imprescindible para la investigación conocer sus percepciones, vivencias y sus experiencias inherentes a la naturaleza de su labor docente. Así mismo, es de señalar que la intencionalidad del estudio fue enfocada como una manera de buscar y presentar respuestas a la problemática en estudio como también a la calidad del proceso de enseñanza-aprendizaje que reciben los alumnos.

Por otro lado, los resultados que se derivan del análisis sobre la información registrada en el capítulo al cual se hace referencia, suministradas por los docentes formadores universitarios permitieron concluir lo siguiente:

1). Predomina una diversidad conceptual acerca del modelo actual de formación integral del estudiante-docente, lo cual demuestra que no hay consenso en relación a lo que cada uno considera respecto a la formación del futuro docente integral; pero a pesar que no existe ese criterio de unificación en cuanto a su formación, la misma es utilizada por cada docente formador desde su propia perspectiva para poder cumplir con el proceso de enseñanzaaprendizaje de los estudiantes-docentes.

2). Hay docentes formadores que se destacan en el trato con los estudiantes por las buenas relaciones que mantienen y la interacción del proceso de formación integral del estudiante-docente, se inscribe en un interesante intercambio de ideas, generando entendimientos, compromisos y respeto en ambos actores del proceso; sin embargo hay otros docentes formadores que deben manejarse entre la tolerancia y el respeto hacia el estudiante-docente para que tenga lugar el proceso de enseñanza-aprendizaje que se espera lograr.

3). Los docentes formadores del subsistema de educación universitaria consideran que tanto el componente de formación pedagógica del estudiante 
docente como el de las prácticas profesionales deberían tener mayor peso durante la formación del docente.

4). Hay una marcada tendencia de la praxis educativa que desarrolla el docente formador universitario, centrada en esquemas rígidos de enseñanza en la cual él es el que sabe y el estudiante-docente de educación integral, se siente imposibilitado para aportar nuevas ideas conducentes a su propio crecimiento tanto personal como a nivel profesional.

5). Se evidencia que la experiencia académica del docente formador de las instituciones universitarias no siempre se corresponde con el nivel de exigencia de las disciplinas que integran el pensum de estudio para la formación del estudiante-docente de educación.

6). A pesar que el docente formador concibe el aprendizaje del estudiante-docente como un proceso activo y participativo, considera que no puede dejar de ser comprensivo y humanizante, pues es necesario conocer cómo piensa, siente y percibe el estudiante su entorno social; aunque también considera que no todos los docentes formadores tienen esa misma visión integradora y significativa durante el proceso de enseñanza que desarrolla.

7). Se pone de manifiesto que el docente formador sigue apegado al modelo de enseñanza-aprendizaje tradicional y no hace esfuerzo alguno en armonizar con la articulación educativa que se debe seguir para estimular la investigación en el estudiante-docente que lo conduzca a la construcción de nuevos conocimientos.

8). Una buena parte de los docentes formadores reconocen que la formación del estudiante-docente, debe ser conducida con una eficacia de alto nivel didáctico y con un dominio de criterios innovador en las diferentes disciplinas impartidas, que su visión formadora debe estar enfocada en la búsqueda de la eficiencia y la calidad de su labor docente, es decir ser un verdadero formador y constructor de aprendizajes que motiven la curiosidad de los educandos para que puedan enfrentar los retos que le depara la vida; 
pero también enfatizan que las instituciones de educación universitarias formadoras de docentes de educación, hagan un esfuerzo mancomunado que esté orientado a rediseñar los planes y programas de estudio para ofrecer una formación integral del estudiante-docente ajustada a las exigencias de las nuevas realidades que hoy perturban el porvenir de las nuevas generaciones tanto en los centros urbanos y en las comunidades rurales.

\section{Referencias}

Balza, A. y Pérez, E., (2009). La formación del docente de educación integral, reflexiones pedagógicas complejizantes para educar la condición humana. Trabajo de investigación científica, UNESR, publicado. Caracas, Venezuela.

Carr, W. y Kemmis, S. (1998). Teoría crítica de la enseñanza. Barcelona, España: Martínez Roca.

Corrales, G. (2008). Formación Docente, Ética y Creatividad. Una Crítica a la Pedagogía de la Certidumbre. Tesis Doctoral. Universidad Pedagógica Libertador. Maracay, Estado Aragua.

Dewey, J. (2007). ¿Cómo pensamos? Relación entre el pensamiento reflexivo y proceso educativo. Madrid: Editorial Paidós.

Fairstein, H. (2003). Pedagogía. Madrid, España: Editorial Calpe.

Martínez, M. (2000). La nueva ciencia, su desafío, lógica y método. México. D.F.: Editorial Trillas.

Márquez Pérez, E (2009). La perspectiva epistemológica cualitativa en la formación de docentes en investigación educativa. Revista de investigación, 66, pp. 13-36.

Morín, E. (2000). La cabeza bien puesta. Repensar la reforma. Reforma el pensamiento. 1ra. Edición. Buenos Aires, República Argentina: Ediciones Visión, SAIC. 
Morín, E. (2001). La cabeza bien puesta. Buenos Aires, República Argentina: Ediciones Visión, SAIC.

Morín, E. (2002). Los siete saberes necesarios para la educación del futuro. Bogotá, D.C., Colombia: Editorial Nomos.

Morín, E. (2003). Introducción al Pensamiento Complejo. Barcelona, España: Editorial Gedisa.

Orozco, L. (2002). Compromiso del docente en la formación integral. Santa Fe de Bogotá, Colombia: Editorial El Búho.

Pérez, A. (2000). Educar valores y el valor de educar. Caracas, Venezuela: Editorial Colección El Simurg.

Rozas, M. (2005). Algunas reflexiones sobre la investigación en el trabajo social en la Argentina. Revistas Temporal, № 74.

Sandín, M. (2003). Investigación Cualitativa en Educación. Fundamentos y Tradiciones. Madrid, España: McGraw and Hill Interamericana.

Savater, F. (2000). El valor de educar. (11a. ed.). Santa Fe de Bogotá, Colombia: Editorial Ariel. S.A.

Ugas, F. (2003). Del acto pedagógico al acontecimiento educativo. San Cristóbal, Táchira, Venezuela: Ediciones del Taller permanente de estudio epistemológico en Ciencias Sociales. 


\section{Naylet Chiquinquirá Rojas Rivero}

e-mail: nayletrojas@hotmail.com; nayletrojas3@gmail.com

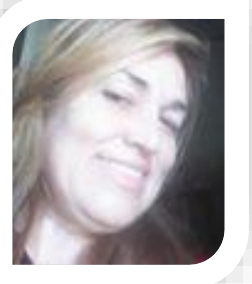

Nací en San Rafael de Onoto del Estado Portuguesa, inserta en el Post Doctorado en estudios libres de la Universidad Fermín Toro-Cabudare Estado Lara (20152016), Doctor en Ciencias de la educación egresada de la Universidad Fermín Toro (2015), Magister Scientiarum en Educación Superior Mención: Docencia Universitaria de la Universidad Fermín Toro (2007), Licenciada en educación Integral área de concentración Ciencias Naturales (2002).

En los actuales momentos, cumplo funciones como docente de aula adscrita a la dirección de educación de la gobernación de Portuguesa con 20 años de servicios. Desde el 2009-2011 cumplió funciones en el departamento de Post grado como coordinadora de la Maestría Educación Superior de la UNEFA Acarigua, paralelo a esos años se desempeñó como docente universitario en la YACAMBU. Araure y La UPEL. Acarigua en las cátedras investigación educativa, investigación aplicada al Derecho, Currículo, Filosofía, entre otras, además es tutora de tesis de pregrado y postgrado, jurado de tesis y participante activa en diversas jornadas de investigación. 